\title{
Fluidic supramolecular nano- and microfibres as molecular rails for regulated movement of nanosubstances
}

\author{
Shun-ichi Tamaru,2, Masato Ikeda', Yusuke Shimidzu', Shinji Matsumoto', Shoji Takeuchi \& Itaru Hamachi¹,4
}

Nano- and micro-sized fibrous architectures are ubiquitous in nature; in particular, microtubules have an essential role within live cells, as tracks for transporting objects to a desired place, driven by molecular motors such as dynein and kinesin. Such functions of bionanofibres motivated us to construct an artificial supramolecular rail using the fluidic property of self-assembled glycolipid nanofibres. Artificial supramolecular nanofibres constructed through molecular selfassembly of small molecules have recently attracted considerable attention for their unique properties, such as reversible formation/destruction under mild conditions and various stimuli responsiveness. In this paper, we show that a supramolecular nanofibre has sufficient fluidity, on the basis of its non-crystalline nature, to function as a molecular track for the directional movement of attached molecules, proteins and nanobeads along the fibre.

\footnotetext{
1 Department of Synthetic Chemistry and Biological Chemistry, Graduate School of Engineering, Kyoto University, Katsura, Kyoto 615-8510, Japan. ${ }^{2}$ Department of Nanoscience, Sojo University, 4-22-1 lkeda, Kumamoto 860-0082, Japan. ${ }^{3}$ Japan Science and Technology Agency (JST), CREST, 5 Sanbancho, Chiyoda-ku, Tokyo 102-0075, Japan. ${ }^{4}$ Center for International Research on MicroMechatronics, Institute of Industrial Science, The University of Tokyo, 4-6-1 Komaba, Meguro-ku, Tokyo 153-8505, Japan. Correspondence and requests for materials should be addressed to I.H. (email: ihamachi@sbchem.kyoto-u.ac.jp).
} 
ubstances having well-defined fibrous nanostructures are anticipated as key materials for developing sophisticated electrical, chemical or biological devices in nanotechnology $^{1-8}$. Carbon nanotubes ${ }^{9}$ are a representative fibrous nanomaterial, not only in nanoelectronic devices such as an electric wire, a capacitor, a transistor or an emitter of field emission display but also in nanomechanics as a probe for a scanning tunneling microscope or atomic force microscope ${ }^{10}$. As alternatives to the carbon nanotube, self-assembled fibrous architectures have recently been developed by bottom-up technology. For instance, a nanotube comprising an amphiphilic hexabenzocoronene derivative was reported to show high electric conductivity through a crystalline $\pi$-stacked graphitic layer ${ }^{7,8}$. Supramolecular nanofibres were also elegantly used as templates for synthesis of inorganic nanomaterials ${ }^{11,12}$. Apart from the use of crystalline nanofibres, dynamic and flexible properties such as fluidity should be one of the unique and essential features of supramolecular fibres, although these have been insufficiently explored. In nature, indeed, flexible selfassembled bionanofibrils have important roles for in-cell molecular transportation systems, cell migration and other dynamic phenomena ${ }^{13}$. For example, natural motor proteins such as myosin, kinesin or dynein walk along bionanofibrils (actin filaments or microtubules) unidirectionally, under conditions in which Brownian motion and viscous forces are dominant, using the chemical energy derived from ATP hydrolysis $\left(50-60 \mathrm{~kJ} \mathrm{~mol}^{-1}\right.$ or $\sim 22 k_{B} \mathrm{~T}$, where $k_{B}$ is the Boltzmann constant $)^{14-16}$. Recent studies have emphasized the importance of Brownian motion (diffusive component, such as diffusional search process of the free motor domain head), as well as rigid conformational changes (mechanical component) of motor proteins coupled with ATP hydrolysis for such efficient one-directional transport ${ }^{17-19}$. Very recently, an increasing number of microtubule-binding proteins have been found to exhibit diffusional motility along the microtubule lattice (one-dimensional random walk) ${ }^{20,21}$, which did not require ATP a

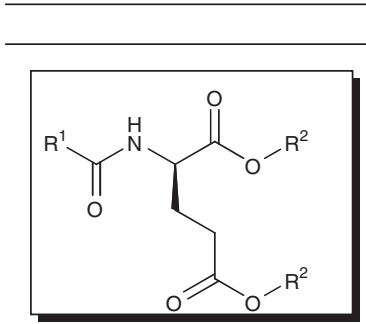

$R^{1}$
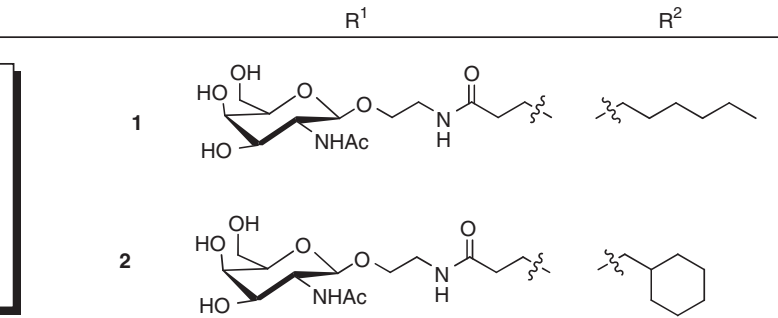

经冾

2

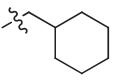

$\mathrm{HO}$<smiles>C=C/C=C/C=C(/C(C)C)N(CC)CC</smiles>

3

$\mathrm{OH}$

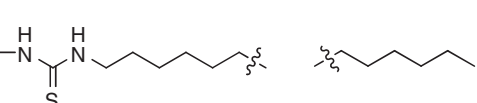

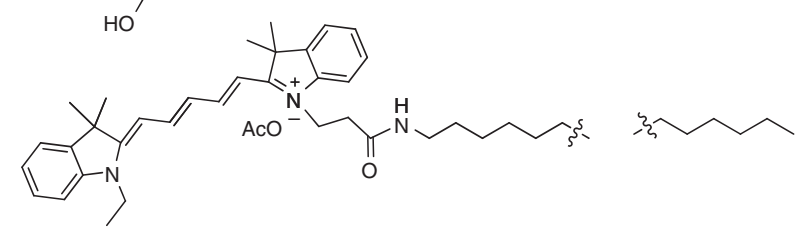

4

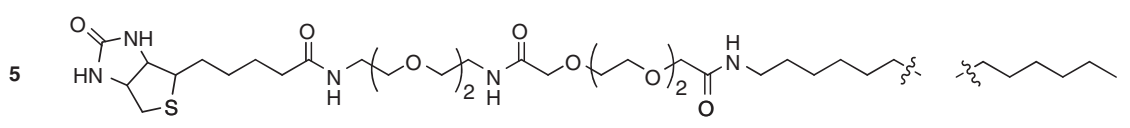

b

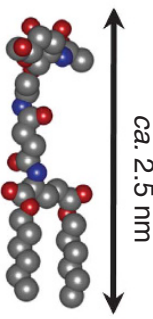

1

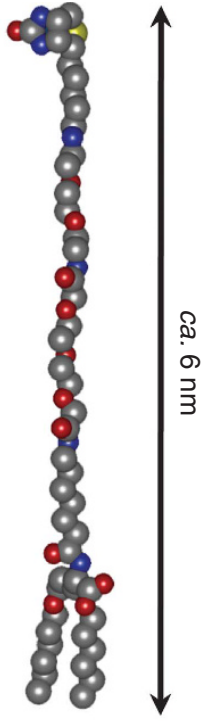

5

C

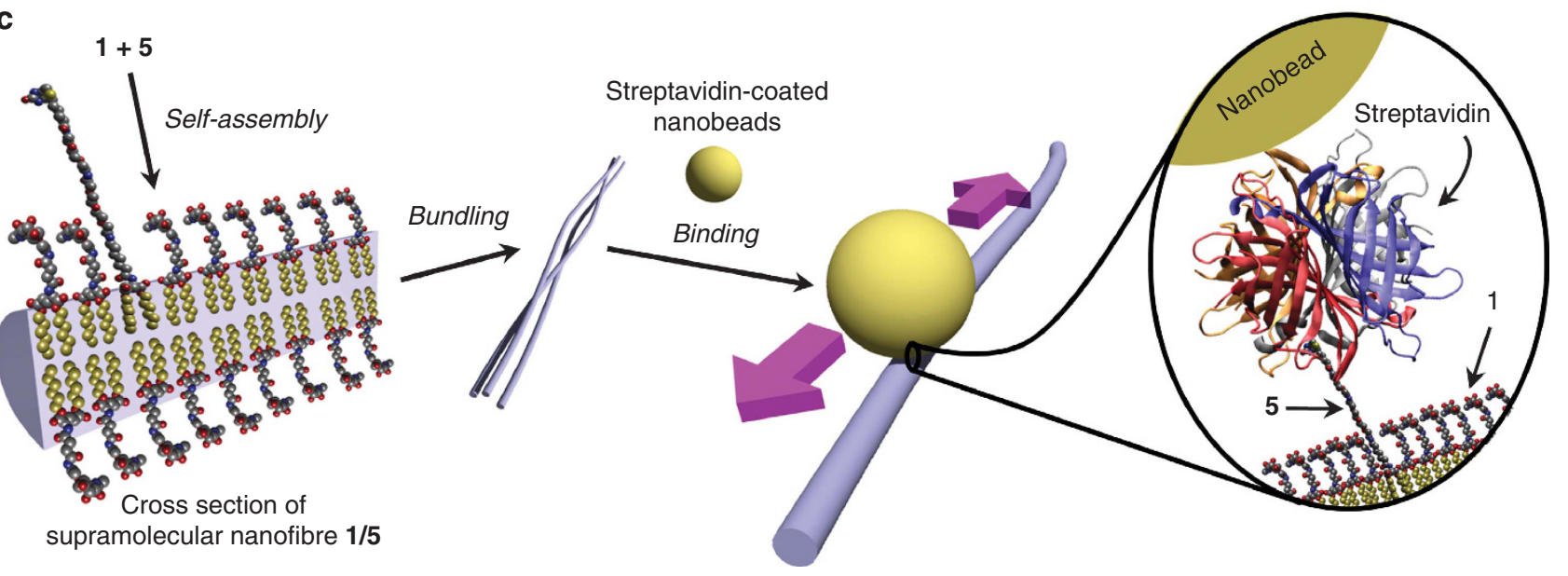

Figure 1 | Supramolecular nanofibre as a molecular rail for nanobeads. (a) Chemical structures of lipid-like molecules 1, 2, FITC-lipid (3), Cy5-lipid (4) and Biotin-lipid (5). (b) Molecular models of lipids $\mathbf{1}$ and 5. (c) Schematic showing self-assembly of lipids $\mathbf{1}$ and $\mathbf{5}$ into a supramolecular nanofibre and binding of nanobeads to the fibre through a biotin-avidin interaction, which results in regulated one-dimensional motion. 
and is thus regarded as an economical mode of transport. Similar one-dimensional diffusive motion without ATP hydrolysis was observed for a DNA-binding protein moving along the DNA fibre ${ }^{22,23}$. However, the proportion and importance of the diffusive component contribution into the one-dimensional transport is still controversial. The construction of simple artificial supramolecular rail-cargo systems on a micrometre scale is expected to provide appropriate models for deep understanding of the role of the diffusive component in one-directional motion and thus give a clue for building a well-developed mass transport network. However, limited knowledge is currently available for artificial supramolecular machinery systems that can work on scales (submicro to micrometre), which are comparable with biological systems ${ }^{24-28}$. In contrast, many elegant synthetic molecular machines have emerged on the nanometre scale, especially in the field of supramolecular chemistry ${ }^{29-38}$.

\section{Results}

Molecular design of supramolecular fibres as molecular rails. We have recently been interested in the hydrogel formation of a self-assembled supramolecular nanofibre in an aqueous solution composed of glycosylated L-glutamate esters bearing two tails, such as $n$-hexyl (1) or methyl-cyclohexyl (2) groups $^{39-42}$ (Fig. 1a). Here, we showed that the gel state is completely distinctive from the crystalline state in terms of fibre fluidity. Using the fluidity, we showed that mixed gelator-like molecules $(\mathbf{3}, \mathbf{4}$ and $\mathbf{5}$, see Supplementary Method) were transported along nanofibre 1. In particular, the biotin-tethered gelator mimic $\mathbf{5}$ was spontaneously mixed to form functionalized nanofibres $\mathbf{1 / 5}$ (Supplementary Fig. S1) that can work as a molecular rail to guide the movements of attached avidin protein and avidin-coated nanobeads in the range of nanometres to tens of micrometres (Fig. 1b,c).

Morphology and fluidic property of supramolecular nanofibres. Transmission and scanning electron microscopic (TEM and SEM) observations of nanofibre $\mathbf{1}$ revealed high-aspect-ratio fibres with a $10-100 \mathrm{~nm}$ range in diameter (Supplementary Fig. S2a,b). A confocal laser-scanning microscopic (CLSM) image also showed fibrous networks stained by 1 -anilinonaphthalene-8-sulphonic acid (ANS), an environmentally sensitive fluorescent probe, suggesting that incipient, thin nanofibres bundled together to form thick microfibres and that the present nano/microfibre contains a well-developed continuous hydrophobic domain (Supplementary Fig. S2c). Interestingly, various morphologies of nanofibre 1 emerged, such as tangled ropes, coiled and wavy fibres (Fig. 2a-c). Moreover, nanofibre 1 can be aligned in a microfluidic channel created by poly(dimethylsiloxane) (PDMS). Figure 2d,e shows the morphological change of nanofibre $\mathbf{1}$ before and after the application of electric voltage $\left(50 \mathrm{~V} \mathrm{~cm}^{-1}\right)$ in a microchannel of PDMS $(150 \mu \mathrm{m}$ in width $\times 50 \mu \mathrm{m}$ in depth), respectively. This result shows that nanofibre 1 has sufficient elastic toughness and flexibility for aligning in microchannels under the application of electric voltage or laminar flow (vide infra).

The fluorescence recovery after photobleaching (FRAP) experiments ${ }^{43}$ gave an insight into the dynamic property of nanofibre 1. Fluorescein isothiocyanate (FITC)-lipid 3 was used as a probe molecule, because FITC derivatives can be easily photobleached by an intense Ar laser light ( $488 \mathrm{~nm}$ ) equipped with CLSM. After the irradiation of the intense laser light to a lariat-shaped nanofibre $\mathbf{1}$, the fluorescence image at the same field of vision was continuously monitored in real time. As shown in Figure 3a, the bleached dark area became bright so that the FITC fluorescence recovered along the original fibre shape within $50 \mathrm{~s}$ because of the rapid supply of non-bleached 3 to the bleached region from the non-bleached edge, which clearly indicates that nanofibre $\mathbf{1}$ possesses fluidity and is structurally continuous at least up to several tens of micrometres. a
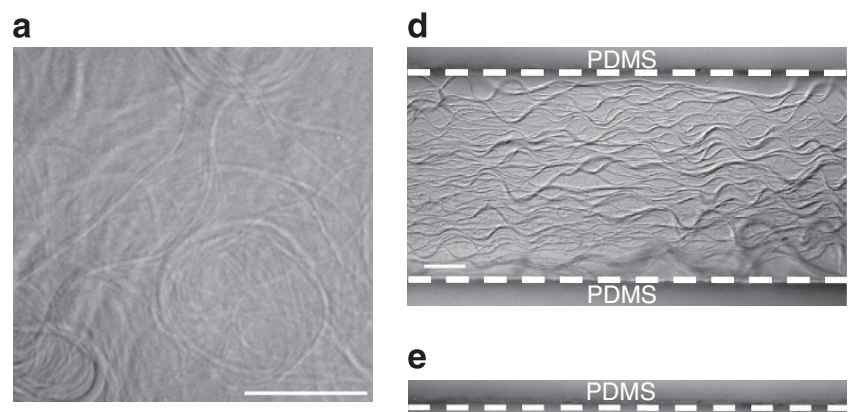

b
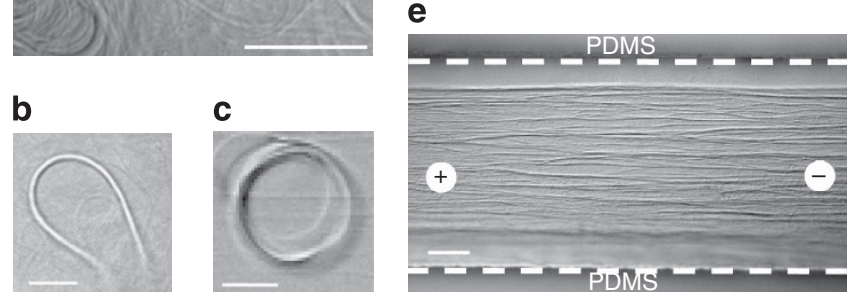

Figure 2 | Morphological variety of supramolecular nanofibre 1

observed by CLSM. (a-c) Various lariat- and coil-shaped nanofibres $\mathbf{1}(\mathbf{1}=0.25 \mathrm{wt} \%(3.8 \mathrm{mM})$ ). (d,e) Nanofibre $\mathbf{1}$ in a microfluidic PDMS (poly (dimethylsiloxane)) device as introduced (d) and linearly aligned by electric field (e) (1=0.25 wt\%, $100 \mathrm{mM}$ Tris(tris(hydroxymethyl)aminomethane)- $\mathrm{HCl}$ buffer ( $\mathrm{pH}$ 7.4)). The fields of vision for images $\mathbf{d}$ and $\mathbf{e}$ were the same and image $\mathbf{d}$ was obtained by applying voltage at $50 \mathrm{~V} \mathrm{~cm}^{-1}$. All images were acquired in differential interference contrast (DIC) mode. The scale bars in a-e are 10, 5, 10, 30 and $30 \mu \mathrm{m}$.

In sharp contrast, such a fluorescence recovery has never been observed in the case of self-assembled crystalline fibre 2 , an analogue of $\mathbf{1}$ bearing more hydrophobic cyclohexyl groups instead of $n$-hexyl groups, indicating that fibre fluidity was completely suppressed in the crystalline state (Fig. $3 b$ ). Figure $3 \mathrm{c}$ shows the typical time profile of the fluorescence recovery process for two different positions on the same fibre (marked as region of interest (ROI) 1 and ROI 2 in Fig. 3c), with a separation of $15 \mu \mathrm{m}$. The fluorescence recovery at ROI 1 was delayed by $18 \mathrm{~s}$ relative to that at ROI 2 , the value of which gave us the lateral diffusion speed $(v)$ of lipids on the supramolecular fibre. The averaged value of lateral diffusion speed $(v)$ was estimated to be $0.7 \pm 0.2 \mu \mathrm{m} \mathrm{s}^{-1}$ from seven independent experimental measurements. It was previously reported that the diffusion coefficient $(D)$ of lipid molecules in biomembranes in aqueous solution at $25^{\circ} \mathrm{C}$ is $\sim 1.0 \mu \mathrm{m}^{2} \mathrm{~s}^{-1}$ (ref. 44 ), which gives a root mean square (r.m.s.) distance $(2 D t)^{1 / 2}=2.0 \mu \mathrm{m}$ during a delay of $1.0 \mathrm{~s}$. Thus, the fluidity of the supramolecular nanofibre $\mathbf{1}$ is slightly lower than that of biomembranes.

Regulated transport of ionic lipids along supramolecular nanofibres. The fluidity of nanofibre $\mathbf{1}$ was further confirmed by an electrically controlled localization change of the ionic probes on it. A cationic fluorescent probe, cyanine 5-lipid 4, was embedded in nanofibre 1, together with anionic FITC-lipid 3. Figure 3d shows typical CLSM images of a coiled fibre during sequential application of electric voltage, in which emissions from $\mathbf{3}$ and $\mathbf{4}$ are coloured green and red, respectively. Without voltage application, the colour of the whole fibre is uniformly yellow, which is a perfectly merged colour of green and red, indicating the homogeneous distribution of $\mathbf{3}$ and $\mathbf{4}$ within the coiled fibre. Under applied voltage, the green emission from 3 gradually moved and concentrated at the cathode (left) side of the fibre, whereas the red emission from 4 concurrently moved to the anode (right) side. After 90 s, these two emission areas were distinctively segregated within different fibre domains, indicating that cationic $\mathbf{4}$ and anionic $\mathbf{3}$ are heterogeneously localized at the anode and cathode sides of the coiled fibre, respectively. The distribution of $\mathbf{3}$ and $\mathbf{4}$ on the fibre was reversibly replaced by one another 
a
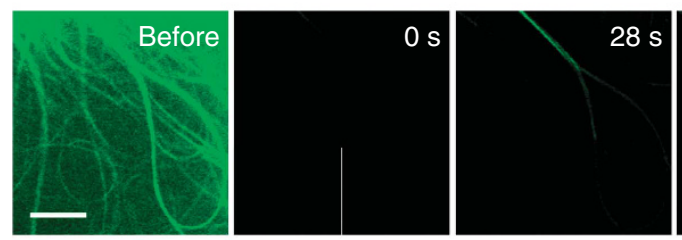

b
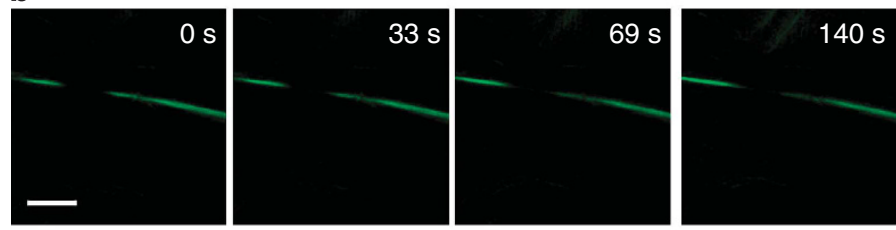

d
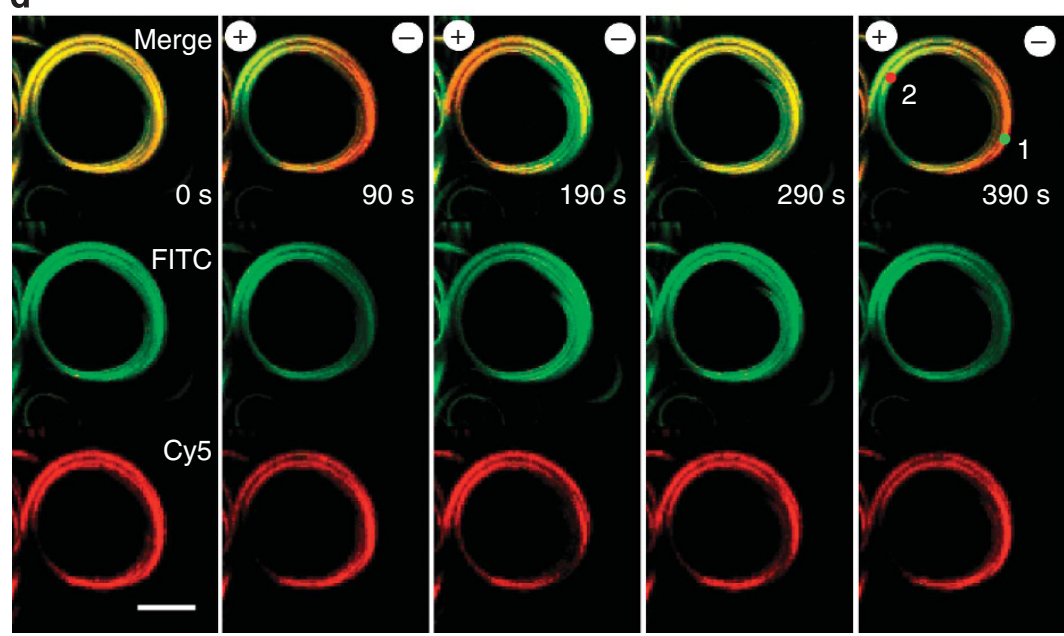

e

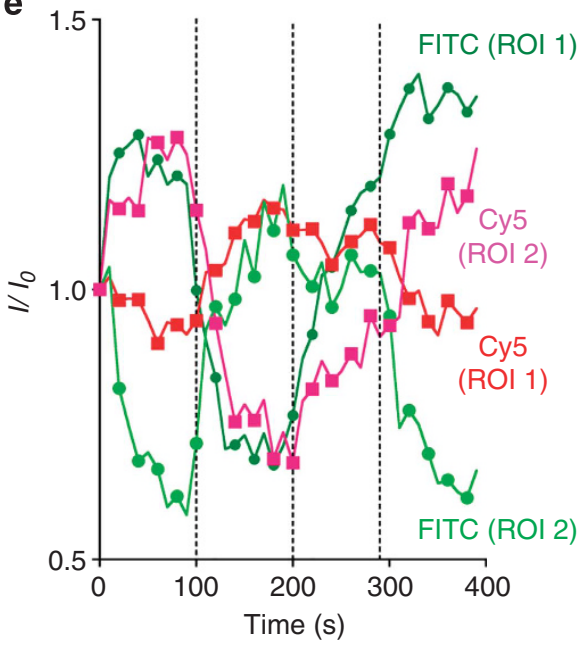

Figure 3 | Fluidic property of supramolecular nanofibre 1 evaluated by FRAP experiment and electrically regulated transport of ionic fluorescent lipids along the fibre. $(\mathbf{a}, \mathbf{b})$ Time-lapse CLSM images for nanofibres $\mathbf{1 / 3}(\mathbf{a})$ and $\mathbf{2 / 3}(\mathbf{b})$ after photobleaching. (c) Plots of fluorescence intensity depending on time for nanofibres $\mathbf{1 / 3}$ at marked regions ROI (region of interest) $\mathbf{1}, \mathbf{2}$ in the inset of $\mathbf{c}$. (1=0.25 wt\%, $\mathbf{2}=0.25 \mathrm{wt} \%$, FITC-lipid $\mathbf{3}=3.0 \mu \mathrm{M}$ in $50 \mathrm{mM}$ HEPES (4-(2-hydroxyethyl)-1-piperazineethanesulfonic acid) buffer ( $\mathrm{pH} 7.2$ )). (d) CLSM images of coil-shaped nanofibres $\mathbf{1} / \mathbf{3}+\mathbf{4}$ during the sequentially applied electric voltage. (e) Plots of fluorescence intensity depending on time at marked ROI 1, 2 shown in the image of $\mathbf{d}(\mathrm{merge}, t=390 \mathrm{~s})(\mathbf{1}=0.25 \mathrm{wt} \%$, agarose $=2.0 \mathrm{wt} \%$, FITC-lipid $\mathbf{3}=3.0 \mu \mathrm{M}$, Cy5-lipid $\mathbf{4}=6.0 \mu \mathrm{M}$ in $50 \mathrm{mM} \mathrm{HEPES} \mathrm{buffer}(\mathrm{pH} 7.2)$; applied voltage, $\left.15 \mathrm{~V} \mathrm{~cm}{ }^{-1}\right)$. Dotted lines denote times when the voltage direction was switched or voltage was cutoff. The scale bars in $\mathbf{a}, \mathbf{b}$ and $\mathbf{d}$ are 10,50 and $10 \mu \mathrm{m}$, respectively.

by switching the direction of the applied voltage (190 s, left: anode, right: cathode), and the biased distribution was readily relaxed by cutting off the voltage (290s). Figure 3e shows the profiles for the fluorescence intensity change of $\mathbf{3}$ and $\mathbf{4}$ at the marked region of the fibre. It is clear that the fluorescence intensity of $\mathbf{3}$ decreased and then increased by switching the voltage direction, the behaviour of which was sharply in contrast to that of $\mathbf{4}$ in each region. These results show that ionic molecules embedded in the supramolecular fibre can be transported along the fibre by an external voltage, depending on the charge of the molecules, because of fibre fluidity.

Regulated motion of nanobeads on supramolecular rails. To trace the motion of a single molecule along the fluidic supramolecular nanofibre directly and individually by microscopy, we next used nanoparticles as probes. Nanoparticle tracking has been recently used to evaluate the motion speed of nanoparticles attached to fluidic membranes ${ }^{45-47}$ or biomolecular motor ${ }^{48,49}$ until hydrodynamic friction against larger nanoparticles set the upper limit for the motion speed. To attach nanoparticles to fibres, we modified the molecular recognition property of supramolecular nanofibre $\mathbf{1}$ with biotin-lipid 5 (Fig. 1c, Supplementary Fig. S1). First, CLSM observations revealed that nanofibres $\mathbf{1 / 5}$ can bind a streptavidin (SA) protein labelled with fluorescence dyes (Supplementary Fig. S3). In addition, the lateral diffusion of the SA protein bound to nanofibres 1/5 was evidenced by FRAP experiments using FITC-labelled SA (Fig. 4a,b, Supplementary Fig. S4), which afforded an averaged diffusion coefficient $(D)$ of $0.12 \pm 0.03 \mu \mathrm{m}^{2} \mathrm{~s}^{-1}$ at $25^{\circ} \mathrm{C}$. From the diffusion coefficient, the r.m.s. distance $(2 D t)^{1 / 2}$ during a delay of $1.0 \mathrm{~s}$ was estimated to be $0.5 \mu \mathrm{m}$, which is comparable with the value of the lateral diffusion speed of lipids on supramolecular fibre 1 . In addition, the FRAP recovery percentage reached $\sim 90 \%$ (Fig. 4b). These data indicate that the binding of SA to biotin-lipid 5 in supramolecular nanofibre $\mathbf{1}$ did not destroy the continuous structure of the fibres. In contrast, self-assembled fibres $2 / 5$ were able to bind SA but did not show lateral diffusion of the bound SA, which is consistent with the data of Fig. 3a,b (data not shown). We then traced the motion of three different sizes of nanobeads coated with SA (50, 250 and $500 \mathrm{~nm}$ in diameter), which successfully attached to nanofibres 1/5 through an avidin-biotin interaction (Supplementary Figs. S5-7). Most interestingly, by careful examination of the time-lapsed CLSM images and movies, one-dimensional motions of the bound SA beads along the long axis of nanofibres $1 / 5$ were captured at a single molecular level (Fig. 4c, also see Supplementary Movie 1). Such a one-dimensional motion was not observed 

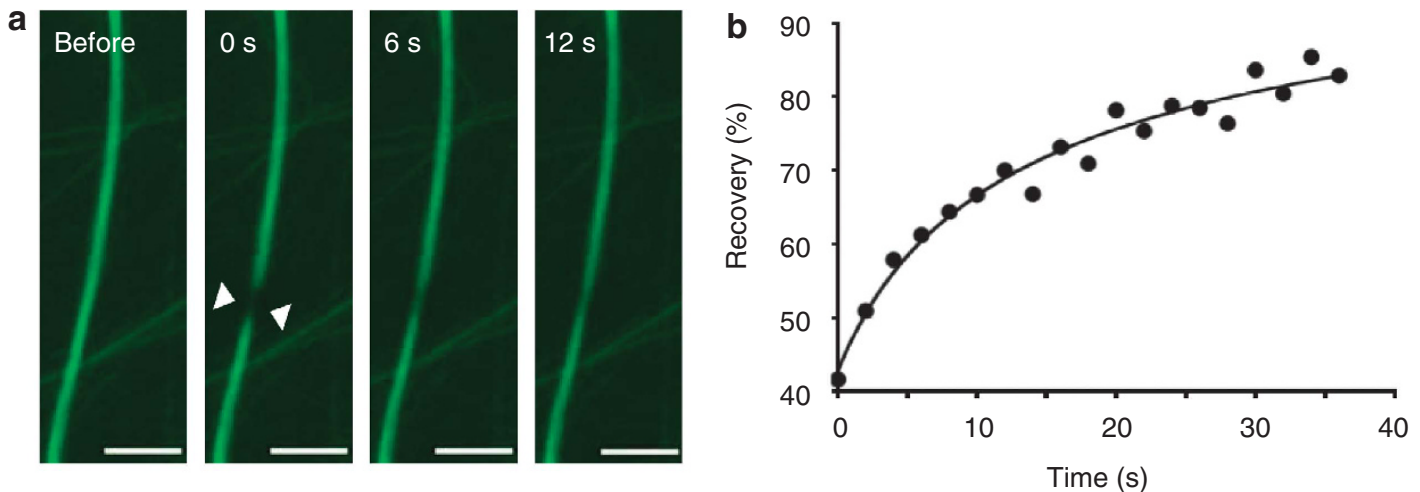

C
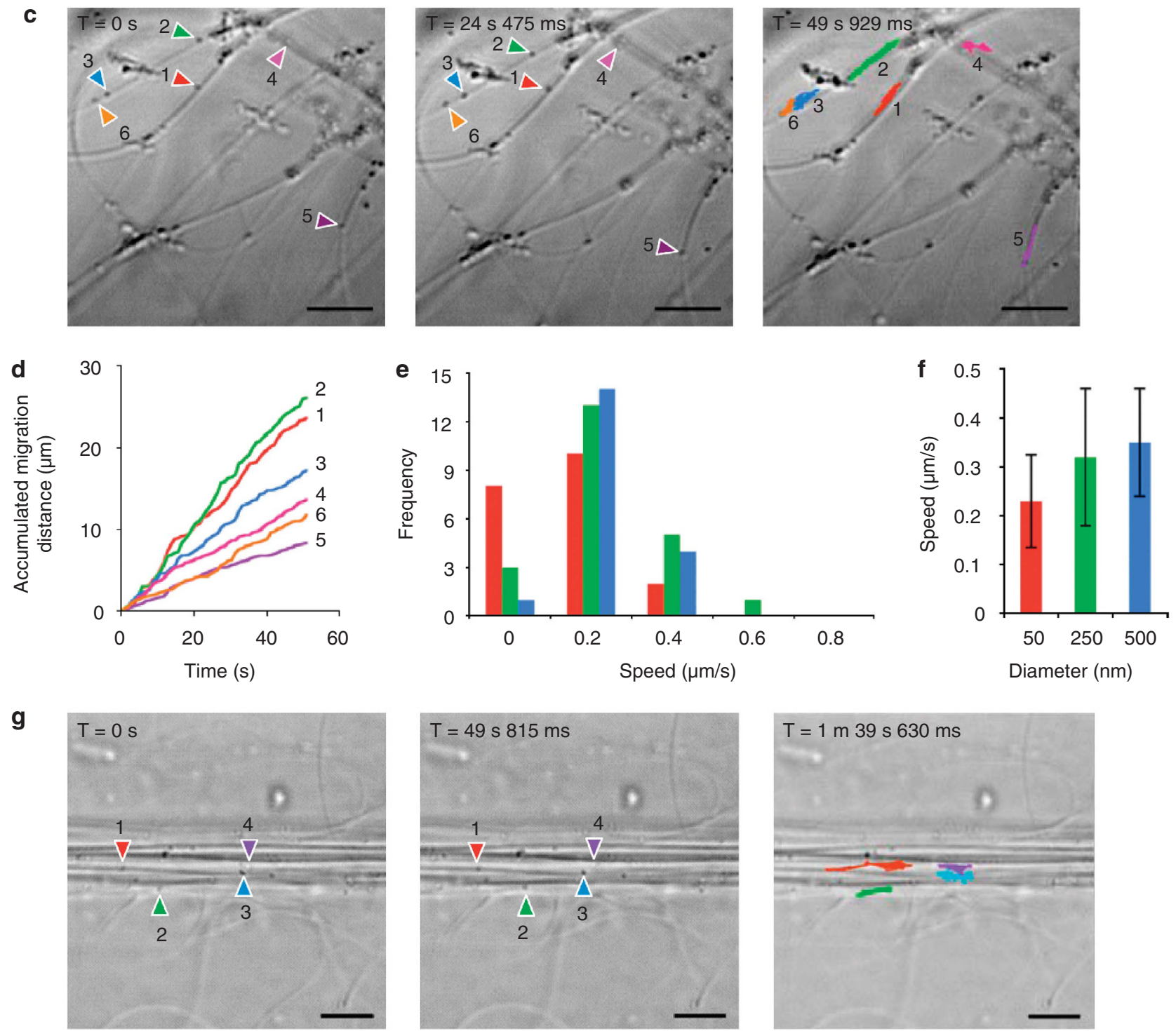

Figure $\mathbf{4}$ | Regulated motions of nanobeads on supramolecular nanofibres 1/5. (a) Time-lapse CLSM images for nanofibres $\mathbf{1 / 5}$ binding FITC-labelled SA after photobleaching. (b) Fluorescence recovery curve with a one-dimensional diffusion model fit (Supplementary Fig. S4) (1=0.10 wt\%, biotin-lipid $\mathbf{5}=24 \mu \mathrm{M}\left(1.6 \mathrm{~mol} \%\right.$ against 1), FITC-labelled SA $=18 \mu \mathrm{g} \mathrm{ml}^{-1}$ ). (c) Time-lapse CLSM images (DIC mode) of nanofibres $\mathbf{1} / \mathbf{5}$ attaching 250-nm SA beads. The trace merged in the right panel denotes the directed motion of the SA beads along the long axis of the nanofibres (Supplementary Movie 1). (d) Time courses of the accumulated migration distances of $250 \mathrm{~nm} \mathrm{SA}$ beads at $25^{\circ} \mathrm{C}$ corresponding to $\mathbf{c}$. (e) Histograms of average lateral diffusion speeds of $50 \mathrm{~nm}$ (red, 20 individual beads), $250 \mathrm{~nm}$ (green, 22 individual beads) and $500 \mathrm{~nm}$ (blue, 19 individual beads) SA beads at $25^{\circ} \mathrm{C}$ evaluated from the series of time-lapse CLSM images (Supplementary Fig. S8). (f) Averaged lateral diffusion speeds depending on the diameter of SA beads. (g) CLSM images (DIC mode) of nanofibres $\mathbf{1 / 5}$ attaching $250 \mathrm{~nm}$ SA beads in a microchannel fabricated by PDMS ( $50 \mu \mathrm{m}$ in height, $300 \mu \mathrm{m}$ in width). The trace merged in the right panel denotes the directed motion of $250 \mathrm{~nm} \mathrm{SA}$ beads along the nanofibres (Supplementary Movie 2) $(\mathbf{1}=0.10 \mathrm{wt} \%$, biotin-lipid $\mathbf{5}=24 \mu \mathrm{M}$ (1.6 mol\% against $\mathbf{1})$ ). The scale bars in $\mathbf{a}, \mathbf{c}$ and $\mathbf{g}$ are $5 \mu \mathrm{m}$. 
for control experiments using nanofibre $\mathbf{1}$ and SA beads (that is, in the absence of 5), or nanofibres 1/5 and SA beads in the presence of excess biotin (that is, with the biotin-binding site of SA masked) (Supplementary Figs. S5-7).

The averaged lateral diffusion speed $(v)$ of the bound beads was estimated to be $0.23 \pm 0.095 \mu \mathrm{m} \mathrm{s}^{-1}$ for $50 \mathrm{~nm}, 0.32 \pm 0.14 \mu \mathrm{m} \mathrm{s}^{-1}$ for $250 \mathrm{~nm}$ and $0.35 \pm 0.11 \mu \mathrm{m} \mathrm{s}^{-1}$ for $500 \mathrm{~nm}$ beads from a plot of accumulated migration distance against time (Fig. 4d), which approximately showed Gaussian distributions (Fig. 4e). The obtained lateral diffusion speeds were almost independent of bead size and were comparable with those of lipid molecules in the nanofibres and the FITC-SA protein bound to the nanofibres (Fig. 4f). These results strongly support the view that the lateral diffusion of beads bound to nanofibre $\mathbf{1}$ is predominantly regulated by the fluidity of the fibre. These values are almost one order of magnitude lower than those for free spheres in water, calculated on the basis of the Stokes-Einstein equation. Moreover, these values are comparable with those for $30 \mathrm{~nm}$ nanogold-coated anti-flourescein $\left(0.26 \mu \mathrm{m}^{2} \mathrm{~s}^{-1}\right)$, which was attached to fluorescein-conjugated phosphatidylethanolamine in a supported bilayer membrane $\mathrm{e}^{45}$, and vesicles $(30-200 \mathrm{~nm}$ in diameter, typically $0.2 \mu \mathrm{m}^{2} \mathrm{~s}^{-1}$ ) attached to a DNA-lipid conjugate by DNA hybridization in a supported bilayer membrane ${ }^{46}$. The latter particles showed two-dimensional random motion on the substrates, which was independent of vesicle size, because of the fluidity of the membrane. In addition, supported bilayer membrane systems require fabricated template materials and tedious procedures under rigidly controlled conditions to construct the one-dimensional systems ${ }^{50-53}$.

By simply aligning nanofibres $\mathbf{1 / 5}$ parallel with the long axis of a microchannel using laminar flow, the motion of the bound nanobeads became directional, in unison along the long axis of the aligned nanofibres 1/5 (Fig. 4g, see also Supplementary Movie 2). The lateral diffusion speed of the bound nanobeads was evaluated to be $\sim 0.3 \mu \mathrm{m} \mathrm{s}^{-1}$, consistent with the bulk data. This result clearly indicates that one can control the orientation of the one-dimensional motion of bound beads by manipulating the alignment of nanofibre 1.

\section{Discussion}

The results presented here show that a fluidic supramolecular nanofibre can function as an artificial molecular rail for converting the three-dimensional random motion of attached substrates such as lipids, proteins and nanobeads into one-dimensional mode. In addition, we can intentionally guide the one-dimensional motion along the aligned fibre in a microchannel because of the elastic toughness and flexibility of the nanofibre. We determined that the averaged speed was $\sim 0.3 \mu \mathrm{m} \mathrm{s}^{-1}$, which is controlled by the lipid mobility in the fluidic fibre, independent of bead size. Interestingly, although the transport mechanism is completely different, this is almost identical to the speed of microtubule-binding protein $^{20}$ and DNA-binding proteins ${ }^{22}$ without ATP energy diffusing along microtubules and DNA $\left(0.1-0.4 \mu \mathrm{m}^{2} \mathrm{~s}^{-1}\right)$, respectively, and is slightly lower than that of kinesin moving on microtubules (about $1 \mu \mathrm{m} \mathrm{s}^{-1}$ ) powered by the energy of ATP hydrolysis ${ }^{54}$. Therefore, it is clear that the present artificial system, which uses lipid fluidity in the nanofibre without chemical energy, can produce a cargo speed comparable with that of biological diffusional motility systems. The directionality of movement cannot be regulated in the present system unlike the one-directional motion of kinesin along microtubules. However, we believe that this is an important first step in the construction of artificial mass transporting systems that can work at the micrometre scale.

\section{Methods}

Supramolecular fibre preparation and CLSM observations. A suspension of glycolipid $\mathbf{1}$ or $\mathbf{2}(1.0 \mathrm{mg}, 0.25 \mathrm{wt} \%)$ in water $(396 \mu \mathrm{l})$ was heated to form a homo- geneous solution. To this solution was added a solution of fluorescent probe (ANS) in methanol and the resulting mixture was gently shaken. The solution $(10 \mu \mathrm{l})$ was spotted on a glass-bottom dish (Matsunami, non-coat, $0.15-0.18 \mathrm{~mm}$ glass bottom), incubated to complete gelation in a sealed box with high humidity at room temperature for $1 \mathrm{~h}$. The sample was then subjected to CLSM observation using an inverted CLSM (Olympus FV1000-ASW, Olympus) equipped with a 543 and $633 \mathrm{~nm}$ Helium Neon, a $488 \mathrm{~nm}$ multi-Ar laser (Coherent). A $\times 100$, NA $=1.40$ oil objective or a $\times 10$, NA $=0.40$ air objective was used to obtain images.

TEM and SEM observations. A suspension of glycolipid $\mathbf{1}(1.0 \mathrm{mg}, 0.25 \mathrm{wt} \%)$ in distilled water $(400 \mu \mathrm{l})$ was heated to form a homogeneous solution and the solution was cooled to room temperature. The solution $(5 \mu \mathrm{l})$ was spotted on a copper TEM grid covered by an elastic carbon-support film $(20-25 \mathrm{~nm})$ with a filter paper underneath, and excess solution was blotted immediately with filter paper. The TEM grid was dried under a reduced pressure for at least $6 \mathrm{~h}$ before TEM observation. No coating or staining was applied for TEM observation. TEM images were acquired using a JEOL JEM-1025 (accelerating voltage, $100 \mathrm{kV}$ ) equipped with a CCD camera. SEM samples were prepared by casting the solution on a silicon wafer and dried under reduced pressure for at least $6 \mathrm{~h}$. Platinum was then sputtered on the dried sample for $90 \mathrm{~s}$ using a JEOL JFC-1600. SEM images were acquired using a JEOL JSM-5600B (accelerating voltage, 15-20 kV) equipped with a CCD camera.

PDMS device fabrication. A mould of PDMS was formed by curing the prepolymer (Sylgard 184; Dow Corning) on a 2 -inch silicon wafer master having a positive relief $(50 \mu \mathrm{m})$ of the capillary channels formed in a photoresist (SU-8) on its surface. The peeled-off PDMS mould was placed on a cover glass (Matsunami, non-coat, $0.15-0.18 \mathrm{~mm}$ glass bottom) and used without permanent bonding.

FRAP study of supramolecular fibres. A glycolipid $\mathbf{1}(1.0 \mathrm{mg})$ was suspended in $50 \mathrm{mM}$ HEPES (4-(2-hydroxyethyl)-1-piperazineethanesulfonic acid) buffer ( $\mathrm{pH}$ $7.2,396 \mu \mathrm{l})$, and the mixture was then heated to form a homogeneous solution. To this solution, a solution of fluorescent probe $(4.0 \mu \mathrm{l})$ in methanol was added and the resulting mixture was gently shaken. The mixture $(10 \mu \mathrm{l})$ was spotted on the cover glass of a dish for CLSM observation and incubated in a sealed box with high humidity at room temperature for $1 \mathrm{~h}$. After a small area of the fibres was viewed on CLSM, the fluorescein moiety of FITC-lipid 3 in this region was photobleached by an intense Ar laser irradiation $(488 \mathrm{~nm})$. The fluorescence image of the same area was continuously monitored in real time using a low level of the monitoring light to suppress further photobleaching. The obtained time-lapse images were analysed with software embedded in a microscope and using Image 1.38 on a Macintosh PC. After importing the data into KaleidaGraph 4.0 (Synergy), each intensity profile was fitted to a Gaussian function and the obtained time decay was curve fitted with a one-dimensional diffusion equation (Fig. 4a,b, Supplementary Fig. S4).

Electrically controlled transport study of supramolecular fibres. In this experiment, the solution of supramolecular nanofibres $\mathbf{1} / \mathbf{3}+\mathbf{4}$ prepared as described above was mixed with agarose $(4 \mathrm{wt} \%)$ to restrict the electrophoretic migration of the nanofibre. The homogeneous mixture containing supramolecular nanofibres $1 / 3+4$ and agarose was introduced into a microchannel (width $\times$ height $=150 \times 5$ $0 \mu \mathrm{m}$ ) of PDMS and the sample was then incubated at room temperature for $1 \mathrm{~h}$. Both ends of the microchannel were sealed by agarose gel to prevent drying and were placed in contact with the electrode. The electric field $\left(15 \mathrm{~V} \mathrm{~cm}^{-1}\right)$ was applied and the movements of the fluorescent probes were traced on CLSM.

Tracing nanobeads attached to supramolecular fibres. A suspension of glycolipid $1(1.0 \mathrm{mg}, 0.10 \mathrm{wt} \%)$ in distilled water $(1.0 \mathrm{ml})$ was heated to form a homogeneous solution. To this warm solution $(50 \mu \mathrm{l})$ was added a DMSO solution $(2.5 \mu \mathrm{l})$ of biotin-lipid $5(0.50 \mathrm{mM})$ and the resulting mixture was gently shaken and incubated at room temperature for $5 \mathrm{~min}$. To this mixture was added a suspension of SA beads $(1.0 \mathrm{wt} \%, 2.0 \mu \mathrm{l})$, and the sample was then subjected to CLSM observation. The trajectories of the beads were tracked with Move-tr/2D 7.0 software (Library) on a Windows PC.

\section{References}

1. Lehn, J.- M. Supramolecular Chemistry: Concepts and Perspectives (VCH, 1995)

2. Shimizu, T., Kogiso, M. \& Masuda, M. Vesicle assembly in microtubes. Nature 383, 487-488 (1996).

3. Oda, R., Huc, I., Schmutz, M., Candau, S. J. \& MacKintosh, F. C. Tuning bilayer twist using chiral counterions. Nature 399, 566-569 (1999).

4. Hirschberg, J. H. et al. Helical self-assembled polymers from cooperative stacking of hydrogen-bonded pairs. Nature 407, 167-170 (2000).

5. Hartgerink, J. D., Beniash, E. \& Stupp, S. I. Self-assembly and mineralization of peptide-amphiphile nanofibers. Science 294, 1684-1688 (2001).

6. de Jong, J. J. D., Lucas, L. N., Kellogg, R. M., van Esch, J. H. \& Feringa, B. L. Reversible optical transcription of supramolecular chirality into molecular chirality. Science 304, 278-281 (2004) 
7. Hill, J. P. et al. Self-assembled hexa-peri-hexabenzocoronene graphitic nanotube. Science 304, 1481-1483 (2004).

8. Yamamoto, Y. et al. Photoconductive coaxial nanotubes of molecularly connected electron donor and acceptor layers. Science 314, 1761-1764 (2006).

9. Iijima, S. Helical microtubules of graphitic carbon. Nature 354, 56-58 (1991).

10. Meyyappan, M. eds. Carbon Nanotubes: Science And Applications (CRC, 2005).

11. Kresge, C. T., Leonowicz, M. E., Roth, W. J., Vartuli, J. C. \& Beck, J. S. Ordered mesoporous molecular sieves synthesized by a liquid-crystal template mechanism. Nature 359, 710-712 (1992).

12. van Bommel, K. J. C., Friggeri, A. \& Shinkai, S. Organic templates for the generation of inorganic materials. Angew. Chem. Int. Ed. 42, 980-999 (2003).

13. Alberts, B. et al. Molecular Biology of the Cell 4th edn, Chapter 16 (Garland Science, 2002).

14. Ronald, D. V. \& Milligan, R. A. The way things move: looking under the hood of molecular motor proteins. Science 288, 88-95 (2000).

15. Schliwa, M. \& Woehlke, G. Molecular motors. Nature 422, 759-765 (2003).

16. van den Heuvel, M. G. L. \& Dekker, C. Motor proteins at work for nanotechnology. Science 317, 333-336 (2007).

17. Shiroguchi, K. \& Kinoshita, K. Jr. Myosin V walks by lever action and Brownian motion. Science 316, 1208-1212 (2007)

18. Iwaki, M., Iwane, A. H., Shimokawa, T., Cooke, R. \& Yanagida, T. Brownian search-and-catch mechanism for myosin-VI steps. Nat. Chem. Biol. 5, 403-405 (2009).

19. Gennerich, A. \& Vale, R. D. Walking the walk: how kinesin and dynein coordinate their steps. Curr. Opin. Cell Biol. 21, 59-67 (2009).

20. Helenius, J., Brouhard, G., Kalaidzidis, Y., Diez, S. \& Howard, J. The depolymerizaing kinesin MCAK uses lattice diffusion to rapidly target microtubule ends. Nature 441, 115-119 (2006)

21. Cooper, J. R. \& Wordeman, L. The diffusive interaction of microtubule binding proteins. Curr. Opin. Cell Biol. 21, 68-73 (2009).

22. Granéli, A., Yeykal, C. C., Robertson, R. B. \& Greene, E. C. Long-distance lateral diffusion of human Rad51 on double-stranded DNA. Proc. Natl. Acad. Sci. USA 103, 1221-1226 (2006).

23. Gorman, J. \& Greene, E. C. Visualizing one-dimensional diffusion of proteins along DNA. Nat. Struct. Mol. Biol. 15, 768-774 (2008).

24. Tabe, Y. \& Yokoyama, H. Coherent collective precession of molecular rotors with chiral propellers. Nat. Mater. 2, 806-809 (2003).

25. Vicario, J. et al. Catalytic molecular motors: fuelling autonomous movement by a surface bound synthetic manganese catalase. Chem. Commun. 2005, 3936-3938.

26. Berná, J. et al. Macroscopic transport by synthetic molecular machines. Nat. Mater. 4, 704-710 (2005).

27. Eelkema, R. et al. Nanomotor rotates microscale objects. Nature 440, 163-163 (2006).

28. Kobatake, S., Takami, S., Muto, H., Ishikawa, T. \& Irie, M. Rapid and reversible shape changes of molecular crystals on photoirradiation. Nature 446, 778-781 (2007).

29. Balzani, V., Credi, A., Raymo, F. M. \& Stoddart, J. F. Artificial molecular machines. Angew. Chem. Int. Ed. 39, 3348-3391 (2000).

30. Balzani, V., Venturi, M. \& Credi, A. Molecular Devices and Machines (WILEY$\mathrm{VCH}, 2003)$.

31. Browne, W. R. \& Feringa, B. L. Making molecular machine work. Nat. Nanotech. 1, 25-35 (2006).

32. Kay, E. R., Leigh, D. A. \& Zerbetto, F. Synthetic molecular motors and mechanical machines. Angew. Chem. Int. Ed. 46, 72-191 (2007).

33. Shinkai, S., Ogawa, T., Nakaji, T., Kusano, Y. \& Manabe, O. Photocontrolled extraction ability of azobenzene-bridged azacrown ether. Tetrahedron Lett. 20 , 4569-4572 (1979).

34. Shigekawa, H., Miyake, K., Sumaoka, J., Harada, A. \& Komiyama, M. The molecular abacus: STM manipulation of cyclodextrin necklace. J. Am. Chem. Soc. 122, 5411-5412 (2000).

35. von Delius, M., Geertsema, E. M. \& Leigh, D. A. A synthetic small molecule that can walk down a track. Nat. Chem. 2, 96-101 (2010).

36. Thordarson, P., Bijsterveld, E. J. A., Rowan, A. E. \& Nolte, R. J. M. Epoxidation of polybutadiene by a topologically linked catalyst. Nature 424, 915-918 (2003).

37. Hiraoka, S., Shiro, M. \& Shionoya, M. Heterotopic assemblage of two different disk-shaped ligands through trinuclear silver(I) complexation: ligand exchange-driven molecular motion. J. Am. Chem. Soc. 126, 1214-1218 (2004).
38. Muraoka, T., Kinbara, K. \& Aida, T. Mechanical twisting of a guest by a photoresponsive host. Nature 440, 512-515 (2006).

39. Kiyonaka, S., Sugiyasu, K., Shinkai, S. \& Hamachi, I. First thermally responsive supramolecular polymer based on glycosylated amino acid. J. Am. Chem. Soc. 124, 10954-10955 (2002)

40. Kiyonaka, S. et al. Semi-wet peptide/protein array using supramolecular hydrogel. Nat. Mater. 3, 58-64 (2004).

41. Yamaguchi, S. et al. Thermally responsive supramolecular nanomeshes for on/off switching of the rotary motion of $\mathrm{F}_{1}$-ATPase at the single-molecule level. Chem. Eur. J. 14, 1891-1896 (2008).

42. Matsumoto, S. et al. Photo gel-sol/sol-gel transition and its patterning of a supramolecular hydrogel as stimuli-responsive biomaterials. Chem. Eur. J. 14, 3977-3986 (2008).

43. Thompson, N. L., Burghardt, T. P. \& Axelrod, D. Measuring surface dynamics of biomolecules by total internal reflection fluorescence with photobleaching recovery or correlation spectroscopy. Biophys. J. 33, 435-454 (1981).

44. Alberts, B. et al. Molecular Biology of the Cell 4th edn, pp 587 (Garland Science, 2002).

45. Lee, G. M., Ishihara, A. \& Jacobson, K. A. Direct observation of Brownian motion of lipids in a membrane. Proc. Natl. Acad. Sci. USA 88, 6274-6278 (1991).

46. Yoshina-Ishii, C. et al. Diffusive dynamics of vesicles tethered to a fluid supported bilayer by single-particle tracking. Langmuir 22, 5682-5689 (2006).

47. Saxton, M. J. \& Jacobson, K. Single-particle tracking: applications to membrane dynamics. Ann. Rev. Biophys. Biomol. Struct. 26, 373-399 (1997).

48. Sasaki, N. et al. One rotary mechnism for $\mathrm{F}_{1}-\mathrm{ATPase}$ over ATP concentrations from millimolar down to nanomolar. Biophys. J. 88, 2047-2056 (2005).

49. Yasuda, R., Noji, H., Kinosita, K. Jr. \& Yoshida, M. F-ATPase is a highly efficient molecular motor that rotates with discrete $120^{\circ}$ steps. Cell 93, $1117-1124$ (1998).

50. Groves, J. T., Ulman, N. \& Boxer, S. G. Micropatterning fluid lipid bilayers on solid supports. Science 275, 651-653 (1997).

51. Artyukhin, A. B., Shestakov, A., Harper, J., Bakajin, O., Stroeve, P. \& Noy, A. Functional one-dimensional lipid bilayers on carbon nanotube templetes. J. Am. Chem. Soc. 127, 7538-7542 (2005).

52. Huang, S.-H. J. et al. Formation, stability, and mobility of one-dimensional lipid bilayers on polysilicon nanowires. Nano Lett. 7, 3355-3359 (2007).

53. Sirbuly, D. J. et al. Biofunctional subwavelength optical waveguides for biodetection. ACS Nano 2, 255-262 (2008).

54. Schnitzer, M. J. \& Block, S. M. Kinesin hydrolyses one ATP per 8-nm step. Nature 388, 386-390 (1997).

\section{Acknowledgments}

We thank Dr D Itoh and Dr R Gojo (Tokyo University) for their kind help in creating silicon wafer masters for PDMS devices, Dr K Kuwata (Kyoto University) for HRMS measurements, Dr A Narita (Kyoto University) for her kind support in using TEM. We gratefully acknowledge the financial support from the JST (Japan Science and Technology Agency), the CREST program and the global COE program, 'Integrated Materials Science' of the Ministry of Education, Culture, Science, Sports and Technology (Japan).

\section{Author contributions}

I.H. conceived the project. S.-i.T., M.I., S.M., S.T. and I.H. designed the experiments. S.-i.T., M.I. and Y.S. performed the experiments. The paper was written by S.-i.T., M.I and I.H. and edited by all the co-authors.

\section{Additional information}

Supplementary Information accompanies this paper on www.nature.com/ naturecommunications

Competing financial interests: Authors declare they have no competing financial interests.

Reprints and permission information is available online at http://npg.nature.com/ reprintsandpermissions/

How to cite this article: Tamaru, S.-i. et al. Fluidic supramolecular nano- and microfibers as molecular rails for regulated movement of nanosubstances. Nat. Commun. 1:20 doi: 10.1038/ncomms1018 (2010). 